OPEN ACCESS

Edited by:

Pietro Pietrini,

IMT School for Advanced Studies

Lucca, Italy

Reviewed by:

Frauke Nees,

Central Institute of Mental Health,

Germany

Francesca Benuzzi

University of Modena and Reggio

Emilia, Italy

${ }^{*}$ Correspondence:

Yawei Cheng

ywcheng2@ym.edu.tw

tThese authors have contributed equally to this work.

Received: 11 April 2017 Accepted: 21 August 2017 Published: 04 September 2017

Citation:

Cheng Y, Chen C and Decety J (2017) How Situational Context Impacts Empathic Responses and Brain Activation Patterns Front. Behav. Neurosci. 11:165. doi: 10.3389/fnbeh.2017.00165

\section{How Situational Context Impacts Empathic Responses and Brain Activation Patterns}

\author{
Yawei Cheng ${ }^{1,2 * t}$, Chenyi Chen ${ }^{1 \dagger}$ and Jean Decety ${ }^{3}$ \\ ${ }^{1}$ Institute of Neuroscience, National Yang-Ming University, Taipei, Taiwan, ${ }^{2}$ Department of Physical Medicine and \\ Rehabilitation, National Yang-Ming University Hospital, Yilan, Taiwan, ${ }^{3}$ Department of Psychology and Department of \\ Psychiatry and Behavioral Neuroscience, University of Chicago, Chicago, IL, United States
}

Clinical empathy, which is defined as the ability to understand the patient's experience and feelings from the patient's perspective, is acknowledged to be an important aspect of quality healthcare. However, how work experience modulates the empathic responses and brain activation patterns in medical professions remains elusive. This fMRI study recruited one hundred female nurses, who varied the length of work experience, and examined how their neural response, functional connectivity, and subjective evaluations of valence and arousal to perceiving another individual in physical pain are modulated by the situational context in which they occur (i.e., in a hospital or at home). Participants with longer hospital terms evaluated pain as less negative in valence and arousal when occurring in a hospital context, but not in a home context. Physical pain perceived in a hospital compared to a home context produced stronger activity in the right temporoparietal junction (rTPJ). The reverse comparison resulted in an increased activity in the insula and anterior midcingulate cortex (aMCC). Mediation analysis indicated that reduced personal accomplishment, a symptom of burnout, breaks down the mediation effect of the putamen on context-dependent valence ratings. Overall, the study demonstrates how situational contexts significantly influence individuals' empathic processing, and that perceiving reward from patient care protects them from burnout.

\section{Highlights}

- Differences in behavior ratings and brain activations between medical practitioners perceiving others' pain in a hospital and at home.

- Situational contexts significantly influence individual's empathic processing.

- Perceiving rewards from patient care protects medical practitioners from burnout.

- Empathy is a flexible phenomenon.

Keywords: clinical empathy, emotional regulation, functional MRI, situational context, burnout

\section{INTRODUCTION}

Empathy, the ability to recognize and share the feelings of others, appears to be a situated cognitive process, embedded with specific contextual cues that trigger different automatic and controlled responses (Decety and Jackson, 2004; Melloni et al., 2014). While empathy clearly has a positive role in social interaction, excessive levels of emotional sensitivity may lead to negative outcomes 
such as personal distress, burnout or desensitization. One apt example is clinical empathy. In the context of caregiving environments, medical practitioners, such as physicians, emergency workers and nurses, have no choice but to deal with people who are suffering or traumatized as part of their everyday routine. How these practitioners respond to, and experience this suffering can have a significant impact on their own well-being (Figley, 2012; Gleichgerrcht and Decety, 2012; Halpern, 2012). Yet, their responses to others' suffering may be situationdependent, and drastically vary across social contexts. This view aligns with the second-person approach to social cognition, which suggests that interpersonal understanding is primarily a matter of social interaction and emotional engagement with others (Baez and Ibanez, 2014; Schilbach, 2014).

Clinical empathy, which is defined as the ability to understand the patient's experience and feelings and view the world from the patient's perspective, is acknowledged to be an important aspect of quality healthcare. An empathetic attitude is associated with improved patient satisfaction, increased adherence to treatment and a positive influence on patients' health (Halpern, 2012). But empathy can also be costly. Medical practitioners are frequently exposed to pain and emotional suffering. Such painful reality may take its toll and lead to compassion fatigue, burnout and professional distress. This can result in a low sense of accomplishment and severe emotional exhaustion (Figley, 2012; Gleichgerrcht and Decety, 2012). Physicians who burn out are more likely to report making medical errors, score lower on instruments measuring empathy, make plans to retire early and experience higher job dissatisfaction. These factors have been linked to a decrease in both patient satisfactions with medical care and patient adherence to treatment plans (Dyrbye and Shanafelt, 2011). Evidence about the interaction between empathy and well-being in medical practitioners seems to be mixed (Zenasni et al., 2012). Some studies, based on self-reported questionnaires, suggest that higher levels of dispositional empathy can be associated with compassion fatigue, exhaustion and burnout (Figley, 1995; Nielsen and Tulinius, 2009). Other studies indicate that empathy protects medical practitioners from burnout (Halpern, 2003). For example, medical practitioners with higher dispositional empathy reported more distress in response to stimuli depicting facial expressions of pain (Gleichgerrcht and Decety, 2014) and more emotional exhaustion, a symptom of burnout (Tei et al., 2014). Therefore, it is no surprise that some authors claim that empathy is always beneficial in medical care and allows physicians to complete clinical tasks more accurately while others argue that keeping an emotional distance from patients maintains clinical neutrality (Riess, 2015).

Neuroscience research can help elucidate the relations between interpersonal sensitivity, empathy, situational contexts and caregiving, and ultimately help physicians to maintain high levels of empathy in clinical practice (Decety et al., 2014). One study reported that higher level of emotional exhaustion in nurses is associated with lower activation in the insula and right temporoparietal junction (rTPJ; Tei et al., 2014), a region that plays an important role in self-other distinction and the sense of agency (Silani et al., 2013). Physicians who report higher perspective-taking abilities derive higher satisfaction from perceived treatment success. These physicians showed stronger activation in the rostral part of the anterior cingulate cortex (ACC), a region associated with reward processing, when viewing patient-physician interactions during which the patient was experiencing pain (Jensen et al., 2014).

Another important issue in clinical empathy is that repeated exposure to patients' suffering may lead to desensitization, which in turn may hamper medical practitioners' motivation to detect the pain of another person (Franck and Bruce, 2009). Neuroimaging research has reliably documented that perceiving other people in physical or emotional pain elicits neurohemodynamic responses in a specific brain network, including the anterior insula, anterior midcingulate cortex (aMCC), somatosensory cortex (SI/II) and brainstem (Jackson et al., 2006; Lamm et al., 2011). Because these areas belong to the so-called "pain-matrix" (i.e., regions consistently activated by acute physical pain), this overlap between the perception of pain in others and first-hand experience of pain has been interpreted as a neural mechanism by which one may share the pain of another (Decety, 2011; Chen et al., 2012). Activation in that network is modulated by interpersonal relationships, group preferences and attitudes (Decety et al., 2010a; Morrison et al., 2012). However, recent studies challenge this interpretation by showing that activity in the pain-matrix may better be interpreted as increased saliency and relevance to pain-related cues rather than actual empathic processing (Decety, 2015). In support of such an interpretation, prior exposure to stimuli depicting somatic pain did not increase the neural response in the pain matrix, but instead led to decreased activities in these regions (Preis et al., 2013).

Some studies have also reported that medical practitioners are less accurate in identifying painful expressions (Xavier Balda et al., 2000; Kappesser and Williams, 2002). One previous neuroimaging study examined neurohemodynamic responses in physicians and non-physician controls when they viewed short video clips depicting hands and feet being pricked by a needle (painful situations) or being touched by a Q-tip (non-painful situations; Cheng et al., 2007). An increased activation in the pain network was detected in the non-physicians when they attended to the painful situations relative to the non-painful ones. A strikingly different pattern was found in the physicians. The brain regions underpinning executive function, self-regulation (dorsolateral and medial prefrontal cortex, dlPFC/dmPFC) and attention (superior parietal cortex and rTPJ) was activated, rather than the pain network. A follow-up study recorded event-related potentials in physicians when they were presented with the same visual stimuli (Decety et al., 2010b). The results showed an early N110 and a late P3 in the control participants. In contrast, no such early and late ERP responses were detected in the physicians, which indicates that affect regulation has very early effects, inhibiting the bottom-up processing that may lead to negative arousal arising from the perception of painful stimuli.

The desensitization or reduced arousal to others' pain could be a double-edged sword to medical professions. On the one 
hand, it could help clinical practitioners to minimize personal distress, combing with elevated brain activities in the regions underpinning executive function, self-regulation and attention, while facing the enormous pain that was expressed by the patients, to provide treatment appropriately. On the other hand, it might also hamper medical practitioners' motivation to detect the pain of another person.

It remains elusive from the existing neuroscience literatures whether and how work experience impacts changes in behavioral and brain responses to the pain of others in medical practitioners. Furthermore, no study has yet addressed the role of situational context in modulation of these responses in this population. Based on a second-person approach to social cognition, we hypothesized that work experience would modulate the neurohemodynamic response to perceiving others' pain in nurses only within specific situational context, namely the hospital, where the medical treatment was routinely given. Instead of generalizing the desensitized effect of others' pain to the context outside of work place, the patient healthcare experience should not overall hamper nurses' motivation to detect the pain of another person.

To gain a better understanding of the interaction between work experience, situational context and well-being in medical practitioners, this study employed a well-validated fMRI empathy paradigm (Cheng et al., 2010; Decety et al., 2013) with one hundred nurses to examine how the pattern of brain activation and subjective evaluations of valence and arousal to perceiving another person in physical pain are modulated by the situational context in which they occur (i.e., in a hospital or at home). Given sexual dimorphism of empathy (Yang et al., 2009; Dehning et al., 2012), only female nurses were enrolled. Given that mediation analysis can be used to investigate the role of intermediate variables that lie on the causal path between 2 variables, it provides a descent way to identify the brain mediators of behavioral changes by applying data from functional neuroimaging (Atlas et al., 2010; Lindquist, 2012). Here, we conducted mediation analyses to examine the causal relationship between work experience in healthcare, neural underpinning of emotional and cognitive empathy, and the context-specific modulation on subjective evaluations. Furthermore, how burnout or reward levels moderated the relations between the amount of work experience and neuroempathetic responses were included into analyses (Hasselhorn et al., 2004; Jensen et al., 2014).

\section{MATERIALS AND METHODS}

\section{Participants}

One hundred female nurses were included in this study. All participants were ethnic Chinese aged between 20 years and 55 years. All nurses graduated from a nursing department of a 4-year medical college or 5-year junior college. Their hospital terms ranged from 0.2 to 29.8 years $(M=7.6, \mathrm{SD}=6.3)$. None of the control participants had any healthcare work experience. This study was carried out in accordance with the Declaration of Helsinki. All subjects gave written informed consent for the study, which was approved by the Ethics Committee of National Yang-Ming University Hospital.

\section{Stimuli Selection}

A total of 60 visual stimuli were used to depict injured or uninjured body parts (hands and feet). The stimuli were selected ad hoc for the study from 120 pictures based on the ratings provided by 30 adults ( 15 females, aged $24 \pm 4.5$ years), who did not take part in the fMRI study. Using a 5-point bivariate valence scale (Berntson et al., 2011), 30 pictures were selected with the highest valence ratings $(4.1 \pm 0.8)$ and 30 with the lowest valence ratings $(0.4 \pm 0.6)$ in order to represent the painful and neutral stimuli, respectively. Additionally, 20 pictures were used for priming situational contexts. Ten hospital pictures depicted nurses and doctors working in hospital scenes and 10 pictures were home scene showing family members doing daily activities. The study thus included four categories of stimuli: pain at home context (PHC), neutral at home context (NHC), pain at work context (PWC) and neutral at work context (NWC). For the sake of carefully controlling the validity of the experimental paradigm, we recruited another 25 controls to re-confirm that control participants showed a comparable pattern of neurohemodynamic responses along with many existed literatures that were associated with pain empathy (Supplementary Table S1).

\section{Procedures}

One week before fMRI scanning, each participant took part in the assessment of a number of dispositional measures, including the Interpersonal Reactivity Index (IRI; Davis, 1996), Toronto Alexithymia Scale-Taiwan version (TAS-20-T; Bagby et al., 1994; Lin and Chan, 2003) and Maslach Burnout Inventory Human Service Survey (MBI-HSS; Maslach et al., 1996). Burnout is a multidimensional process with three constructs: emotional exhaustion, depersonalization and reduced accomplishment (Maslach and Jackson, 1981).

The fMRI used a mixed block design (13.2 s ON/17.6 $\pm 7.4 \mathrm{~s}$ OFF; Figure 1A). There were a total of two runs. Each run consisted of eight $\mathrm{ON}$ intermixed with eight OFF blocks. Each ON block consisted of one photo $(2200 \mathrm{~ms})$ for priming and five trials (2200 ms each) from painful or neutral stimuli. To elicit the priming effect of situational context on pain empathy, we showed the picture cue participants about which context to employ at the beginning of each block (at home or in a hospital) and gave the instructions, including the home context ("Imagine that you are facing one person, for example, a friend or family member, in the pain/no-pain at home") and hospital context ("Imagine that you are facing the pain/no-pain with the same person in the hospital"). The OFF condition remaining on screen for $17.6 \mathrm{~s}$ was followed by a jittered fixation cross $(5.0 \pm 4.7 \mathrm{~s})$ presented against a gray background in order to avoid the expectation and multicollinearity among regressors. The order of the stimulus condition was randomized and counterbalanced across runs.

After fMRI scanning, participants were asked to rate the stimuli for their valence and arousal. Participants positioned a cursor on a bivariate display (a $5 \times 5$ grid) in which the horizontal dimension indicated the amount of positive valence and the vertical dimension indicated the amount of negative valence toward these stimuli (Norris et al., 2010). For arousal ratings, participants positioned the cursor on a single dimension display (9-point scale). 


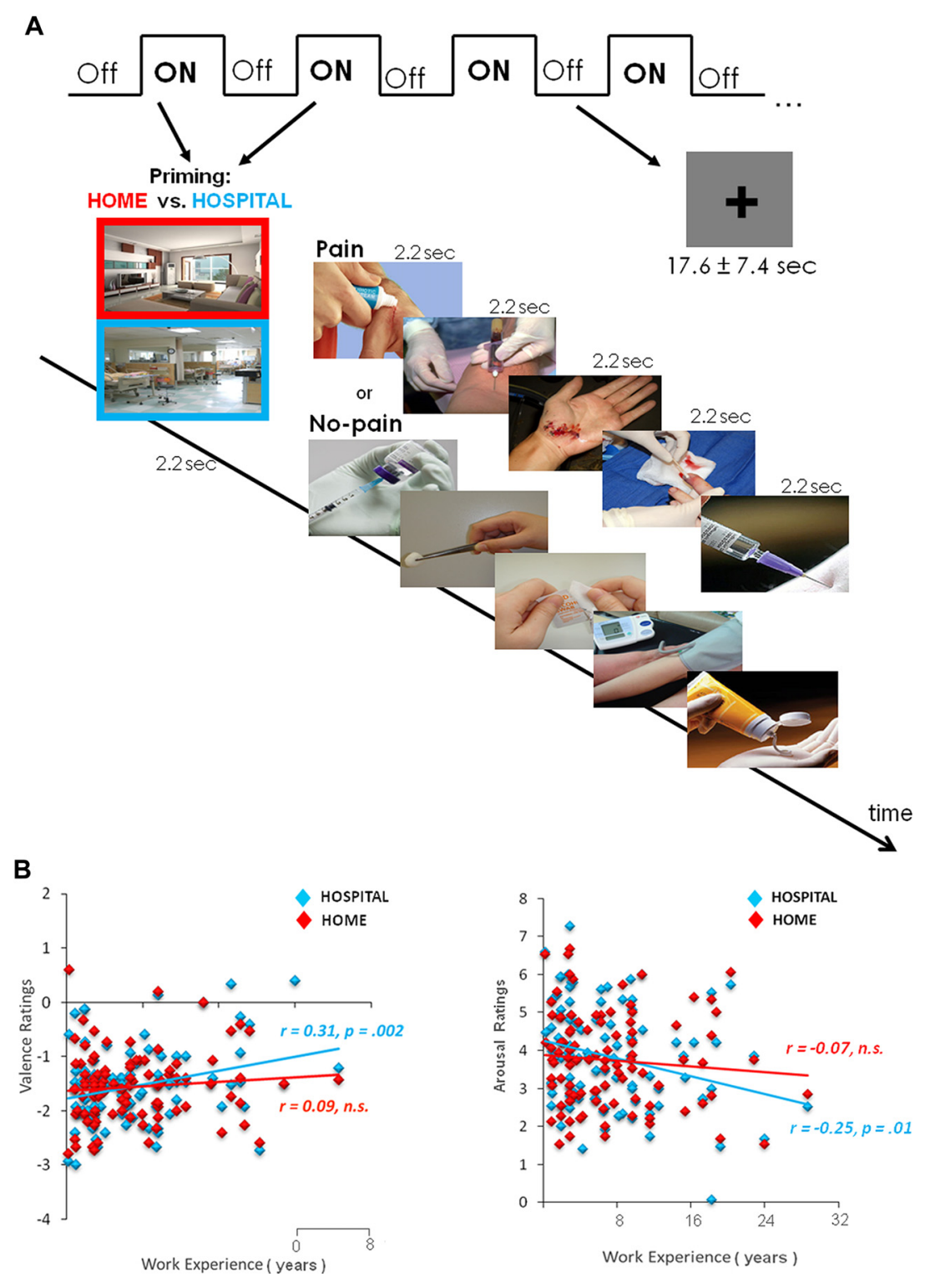

FIGURE 1 | Experimental design and subjective ratings of valence and arousal. (A) The fMRI scanning used a mixed block design. Each block was preceded by one photo for priming the situational context (Home or Hospital). After scanning, participants rated the stimuli in both valence and arousal. (B) Context-dependent valence and arousal ratings of the pain stimuli. Within the hospital context, nurses who had longer hospital terms (years) tended to evaluate the pain stimuli less negatively in both valence and arousal. Fisher r-to-z transformation confirms that the hospital context significantly differed correlation coefficients from the home context $(\Delta z=2.15, p<0.05)$.

\section{Functional MRI Acquisition and Data Analysis}

Images were acquired using a 3T Siemens Magnetom Trio-Tim magnet. For functional images, 3-mm-thick transverse slices oriented along the AC-PC line were continuously collected using an echo-planar imaging sequence $(\mathrm{TR} / \mathrm{RE}=2200 / 30 \mathrm{~ms}$, $\mathrm{FOV}=220 \mathrm{~mm}$, flip angle $=90^{\circ}$, matrix $\left.=64 \times 64\right)$. Functional images were co-registered to the T1 template, and a skullstripped image was created from the segmented gray matter, white matter and CSF images. These segmented images were then combined to create a subject-specific brain template. EPI images were realigned and filtered (128 s cutoff), then co-registered to these brain templates, normalized to MNI space and smoothed (8 mm FWHM).

Functional MRI data were processed with SPM8 (Wellcome Department of Imaging Neuroscience, London, UK) in MATLAB 7.0 (MathWorks Inc., Sherborn, MA, USA). Data were entered into a general linear model, with movement parameters as nuisance regressors. A two-stage general linear model was used to examine the effect sizes of each condition. 
At the first level analysis, four conditions (PHC, NHC, PWC, NWC) were modeled separately with a duration of $13.2 \mathrm{~s}$ beginning at the onset of the priming photo. The null event (fixation) was modeled with the duration $17.6 \pm 7.4$ s. Linear contrasts were applied to obtain parameter estimates. At the second-level analysis, images of parameter estimates from the first level were collapsed into a $2 \times 2$ factorial design with stimulus (Pain vs. Neutral) and context (Work vs. Home) as the within-subject variables. Groupwise effects for the following contrasts were corrected for multiple comparisons family-wise error rate (FWE) of $p<0.05$ (cluster size $k>20$ ). To explore the extent to which context-dependent hemodynamic changes found in the group-wise analysis were related to subjective evaluations, whole brain correlation analyses were conducted with valence and arousal ratings in the home or hospital context, respectively (thresholded at $p<0.001$, and cluster extent of at least 20 contiguous voxels, FWE at $p<0.05$ ).

In addition, valence and arousal ratings were entered as covariates and thresholded at an uncorrected $p<0.001$ with a cluster extent of at least 25 contiguous voxels (FWE of $p<0.05$ ). The MarsBar toolbox was used to extract the mean activity within a 6 -mm-radius sphere (FWE-corrected, $p<0.05$ ) from pre-defined regions of interest (ROIs). The ROI coordinates (rTPJ: 62, -54, 16; right insula: 44, -4, 0; aMCC: 6, 18, 30 in Montreal Neurological Institute (MNI)) were selected from fMRI meta-analyses of theory of mind (ToM) and empathy (Lamm et al., 2011; Bzdok et al., 2013, 2015). The mean activity of each ROI was subjected to repeated measures ANOVA with stimulus (Pain vs. Neutral) and situational context (Work vs. Home) as the within-subject variables.

\section{Functional Connectivity Analysis}

The cluster in the insula as a result of the second level analysis ((PHC - NHC) - (PWC - NWC) ) was selected as a seed given this polysensory region being an integrative hub of the salience network (Menon and Uddin, 2010). Psychophysiological interaction (PPI) was used to investigate task-specific changes in the relationship between hemodynamic responses in different brain areas. Technically, the time series of the first eigenvariates of the BOLD signal were temporally filtered, mean corrected and deconvolved to generate the time series of the neuronal signal for the source region - the insula - as the physiological variable in the PPI. The psychological variable represented the time course of the contrast between painful and non-painful trials. The interaction term in the resulting SPM showed areas with selective connectivity to the insula across the psychological contrast of Pain vs. Neutral. The PPI analysis was performed for each participant, and the resulting images of contrast estimates were entered into a randomeffects group analysis. PPI analyses were separately run for hospital (PWC - NWC) and home (PHC - NHC) contexts to acquire the functional connectivity seeded in the right insula ( $x 44, y-4, z 0)$ between different situational contexts. Results on second-level analysis were reported at a voxel-wise statistical cutoff of $p<0.001$ and a spatial extent threshold of $k>25$ voxels.

\section{Mediation Analysis}

Mediation Effect Parametric Mapping was used to test specific hypotheses about brain-behavior relationships (Baron and Kenny, 1986; Wager et al., 2008). Based on the conceptual framework of a mediation effect (MacKinnon et al., 2007), work experience (hospital terms in years) was selected as the predictor, the context-dependent valence or arousal ratings as the outcome, and the neuro-hemodynamic activity in each ROI as the mediator.

Mediation analyses were conducted in a sequential series of steps. Step 1 identified the context-dependent valence and arousal ratings to pain stimuli. Step 2 used the whole brain correlation analyses to identify the ROIs involved in neuro-empathetic reactions. Step 3 tested whether each ROI mediated the linkage between work experience and context-dependent valence and arousal ratings. Step 4 tested how burnout levels, as indicated by the scores on each MBI-HSS subscale, moderated these existing brain-behavior relations.

Path $a$ coded the link in which the predictor variable must be related to the mediator. Path $b$ coded the link in which the mediator must be directly related to the outcome, controlling for work experience. The mediation effect $\left(a^{*} b\right)$ must be significant, which amounts to a statistical test on the product of the $a$ and $b$ path coefficients. Equivalently, the test for the predictor-outcome relationship would be significantly reduced by the inclusion of the mediator in the path model. We refer the overall predictoroutcome relationship as the $c$ effect, and control the direct effect for the mediator as $c^{\prime}$. The $a^{*} b$ effect was to test the significance of $c-c^{\prime}$.

\section{RESULTS}

\section{Dispositional Measures and Subjective Ratings}

Scores on the depersonalization $(r=-0.21, p=0.039)$ and personal accomplishment subscales $(r=0.23, p=0.021)$ of the MBI-HSS were associated with work experience (years). However, no other dispositional measures showed any correlation.

Subjective valence and arousal ratings of pain stimuli in a hospital context were related to years of work experience (Figure 1B). Nurses with more work experience evaluated pain stimuli as less negative in valence $(r=0.31, p=0.002)$ and arousal $(r=-0.25, p=0.01)$. In contrast, in a home context, work experience was not related to valence $(r=0.09, p>0.1)$ and arousal $(r=-0.07, p>0.1)$. Fisher $r$-to- $z$ transformation $\Delta z=2.15, p<0.05$, confirmed significantly different correlation coefficients between contexts. Direct comparisons between home and hospital contexts on valence $\left(t_{(99)}=0.045, p=0.96\right.$ ) and arousal $\left(t_{(99)}=-1.04, p=0.03\right)$ ratings did not reach significance.

\section{fMRI Group-Wise Comparisons}

For the pain effect ((PHC + PWC) - (NHC + NWC $)$ ), there was significant increment in hemodynamic activity in the 
brain regions involved in pain matrix, including the anterior insula, aMCC, supplementary motor area, inferior frontal gyrus, $\mathrm{dlPFC/dmPFC,} \mathrm{SI/II} \mathrm{and} \mathrm{periaqueductal} \mathrm{gray} \mathrm{(Table} \mathrm{1).}$

To examine the influence of situational context on pain perception, we performed an interaction analysis. When participants perceived painful stimuli within a hospital relative to a home context ((PWC - NWC) vs. (PHC - NHC)), increased activity was detected in the rTPJ. The reversed comparison $((\mathrm{PHC}-\mathrm{NHC})$ vs. (PWC $-\mathrm{NWC}))$ showed increased activity in the insula and aMCC. Results from the ROI analyses are presented in Figure 2.

\section{Functional Connectivity}

Situational contexts elicited distinct patterns in functional coupling when participants viewed painful stimuli. The hospital context, but not the home context, was associated with a positive coupling between the insula and the MCC and a negative coupling with the ventromedial prefrontal cortex (vmPFC; $p<0.001$ and $k>25$ voxels).

\section{Correlations between \\ Neuro-Hemodynamic Activity and \\ Subjective Ratings}

Whole brain correlation analyses showed that, within the home context, valence ratings inversely predicted hemodynamic activity (Pain vs. Neutral) in the insula $(46,6,2 ;-44,4,-4)$. Within the hospital context, more positive valence ratings predicted stronger activities in the putamen $(24,-8,10)$ and dlPFC $(-42,18,30)$. In addition, arousal ratings inversely predicted activities in the ACC $(-14,32,28)$ and dmPFC $(-6,26,52)$. These results were thresholded at individual $p<0.001$, and cluster extent of at least 20 contiguous voxels, FWE at $p<0.05$. The neuro-hemodynamic estimates in these regions were extracted for mediation analyses.

\section{Mediation Analysis Results}

While work experience (hospital terms in years) was selected as the predictor and the context-dependent valence or arousal

TABLE 1 | Pooled group results for all participants $(N=100)$.

\begin{tabular}{|c|c|c|c|c|}
\hline \multirow[t]{2}{*}{ Brain region } & \multicolumn{3}{|c|}{ MNI coordinates } & \multirow[t]{2}{*}{ Peak $T$} \\
\hline & $x$ & $y$ & $z$ & \\
\hline \multicolumn{5}{|l|}{ PAIN > NO-PAIN } \\
\hline - Anterior mid-cingulate cortex & 2 & 22 & 40 & 4.98 \\
\hline - Dorsomedial prefrontal cortex & 0 & 56 & 30 & 4.92 \\
\hline R Anterior insula & 32 & 16 & 0 & 5.08 \\
\hline R Supplementary motor area & 12 & 12 & 72 & 6.11 \\
\hline R Putamen & 30 & -22 & -2 & 4.9 \\
\hline R Supramarginal gyrus & 66 & -18 & 32 & 4.99 \\
\hline R Posterior cingulate cortex & 8 & -38 & 24 & 4.1 \\
\hline L Anterior insula & -40 & 20 & 2 & 4.77 \\
\hline L Inferior frontal gyrus (pars opercularis) & -42 & 6 & 24 & 6.51 \\
\hline L Supplementary motor area & -10 & 18 & 68 & 6.44 \\
\hline LPostcentral gyrus & -58 & -20 & 30 & 4.88 \\
\hline L Ventrolateral prefrontal cortex & -32 & 30 & -8 & 4.81 \\
\hline \multicolumn{5}{|l|}{ HOSPITAL > HOME } \\
\hline L Dorsolateral prefrontal cortex & -50 & -4 & 36 & 4.28 \\
\hline L Postcentral gyrus & -40 & -18 & 38 & 5.52 \\
\hline L Cingulate gyrus & -14 & 4 & 42 & 3.73 \\
\hline L Lingual gyrus & -26 & -64 & -8 & 3.43 \\
\hline R Dorsolateral prefrontal cortex & 50 & -2 & 38 & 4.49 \\
\hline R Temporal pole & 62 & 6 & 2 & 3.92 \\
\hline R Lingual gyrus & 20 & -48 & -8 & 3.41 \\
\hline \multicolumn{5}{|l|}{ (PHC - NHC) vs. (PWC - NWC) } \\
\hline R Insula & 44 & -4 & 0 & 3.87 \\
\hline $\mathrm{R}$ Inferior temporal gyrus & 58 & -20 & -22 & 3.91 \\
\hline
\end{tabular}

All clusters reached significance at family-wise error rate (FWE)-corrected $p<0.05$ (thresholded at $p<0.001$, cutoff, $t=3.11$, uncorrected, a spatial extent threshold $k=20$ ), except those marked by a star, which were taken from pre-defined regions of interest (RO/s) and significant at uncorrected $p<0.05$. 


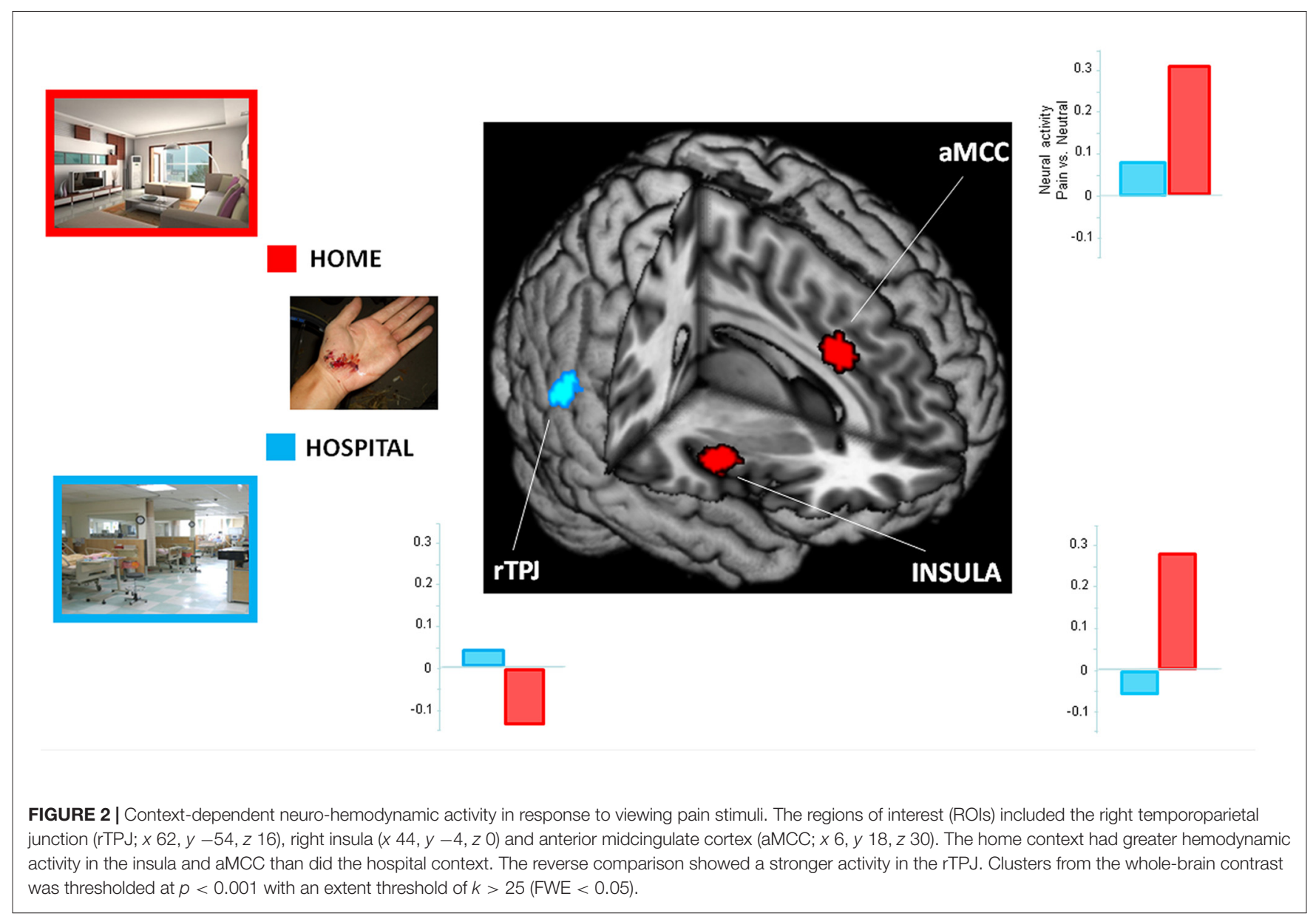

ratings as the outcome, the neuro-hemodynamic activity in each regions, identified by whole brain correlation analyses, was the mediator (Figure 3A). Driven by the mediation model and hypotheses (Figure 3B), we performed analyses to test if each ROI was the mediators of the linkage between work experience and subjective ratings of valence and arousal. Moderation effects of each scale of Burnout were also examined on areas of interest.

\section{Putamen and dIPFC Mediate the Linkage between Work Experience and Context-Dependent Valence Ratings}

Within the hospital context, the hemodynamic activity in the putamen was positively associated with work experience and predicted more positive valence ratings of the pain stimuli: $a=0.001, Z=2.37, b=0.35, Z=2.17$ and $a^{*} b=0.0004, Z=2.22$, all $p<0.05$ (Figure 4). The activity in the dlPFC was positively associated with work experience, and predicted more positive valence ratings: $a=0.003, Z=3.51, b=0.22, Z=2.65$ and $a^{*} b=0.0006, Z=2.82$, all $p<0.05$.

\section{Burnout Moderates the Mediation Effect of Putamen on Context-Dependent Valence Ratings}

Three subscales of MBI-HSS, including emotional exhaustion, depersonalization and reduced personal accomplishment, were included to test if burnout levels affected path $a, b$ and $a^{*} b$. The indirect path $a$ between work experience and putamen activity in the valence model ( $a=0.001, Z=2.41, p=0.016)$ was diminished after the inclusion of scores on reduced personal accomplishment ( $a=8.32 \times 10^{-4}, Z=1.8, p>0.10$ ). The remaining paths were unaffected by other subscales.

\section{ACC and dmPFC Mediate the Linkage between Work Experience and Context-Dependent Arousal Ratings} Within a hospital context, the hemodynamic activity in the ACC was positively associated with work experience, but predicted less arousal ratings of pain stimuli: $a=0.001, Z=3.45, b=-1.04$, $Z=-2.36$ and $a^{*} b=-0.001, Z=-2.09$, all $p<0.05$ (Figure 5). The activity in the dmPFC was positively associated with work experience, but predicted less arousal ratings of the pain stimuli: $a=0.002, Z=2.99, b=-0.59, Z=-2.30$ and $a^{*} b=-0.001$, $Z=-1.96$, all $p<0.05$.

\section{DISCUSSION}

Medical practitioners regularly perceive and react to others' suffering, but this can be costly to their well-being. It is unclear how changes in medical practitioners' behavioral and brain 


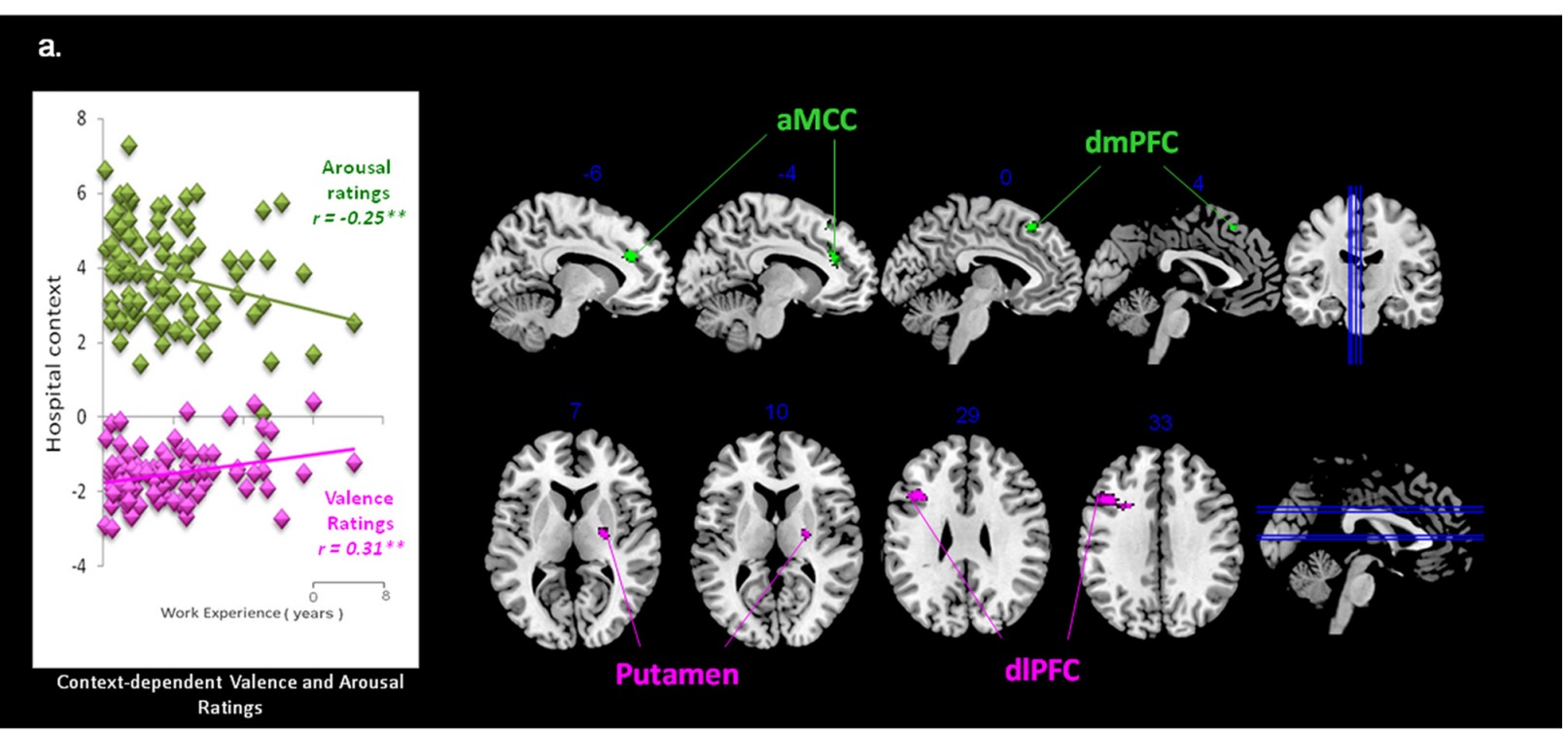

b.

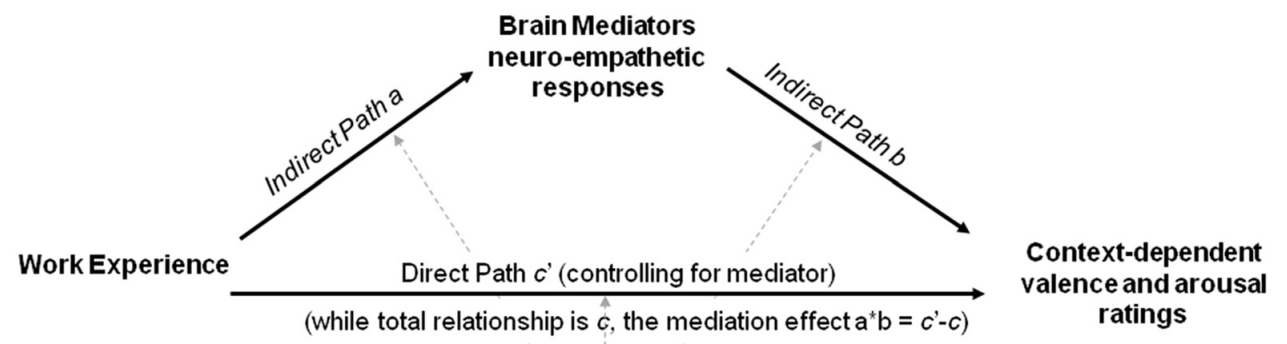

\section{Burnout}

Question 1: Where are the brain mediators of neuro-empathetic responses, mediating the linkage between work experience and context-dependent valence and arousal ratings?

$\begin{array}{ll}\text { Mediation Hypothesis: } & \text { paths } a, b \text { and } a^{*} b \text { were significant. } \\ \text { Direct pathway Hypothesis: } & \text { only direct path } c^{\prime} \text { was significant. }\end{array}$

Question 2: How do burnout levels moderate the relationship?

Moderation Hypothesis: path a, b or c' significantly reduced or vanished after burnout being included into the above statistic model.

Mediation Hypothesis: path $a, b$ or c' significantly reduced and new pathway in connection with burnout emerged after burnout levels were included into this model

Direct pathway Hypothesis: paths $a$, b or c' remained significant and unaffected by the inclusion of burnout.

FIGURE 3 | Hypothesis for mediation analysis. (A) Context-dependent valence and arousal ratings. The correlation between subjective ratings and work experience was specific to the context. Significant correlations occurred between context-dependent subjective ratings and neuro-empathetic reaction (Pain vs. Neutral). Suprathreshold voxels ( $p<0.05$ corrected for voxel-wise comparisons) are displayed across four coronal and sagittal sections on the ch2bet template, using MRIcron software (http://www.sph.sc.edu/comd/rorden/mricron/). The hemodynamic response in the anterior cingulate cortex (ACC; -14, 32, 28) and dorsomedial prefrontal cortex (dmPFC; $-6,26,52$ ) was significantly negatively correlated with arousal ratings (shown in green), whereas the response in the putamen (24, $-8,10)$ and dorsolateral prefrontal cortex (dIPFC; $-42,18,30$ ) was positively correlated with valence ratings (shown in magenta). These areas are the main ROlsfor the mediation analysis. (B) Mediation Model and Hypotheses. While observing the context-dependent valence and arousal ratings, we selected work experience as the predictor variable, and performed analyses to test if the above ROls were the mediators of the linkage between work experience and subjective ratings of valence and arousal. Whether and how each scale of Burnout moderates the existing model and area of interest are considered as well. 
A
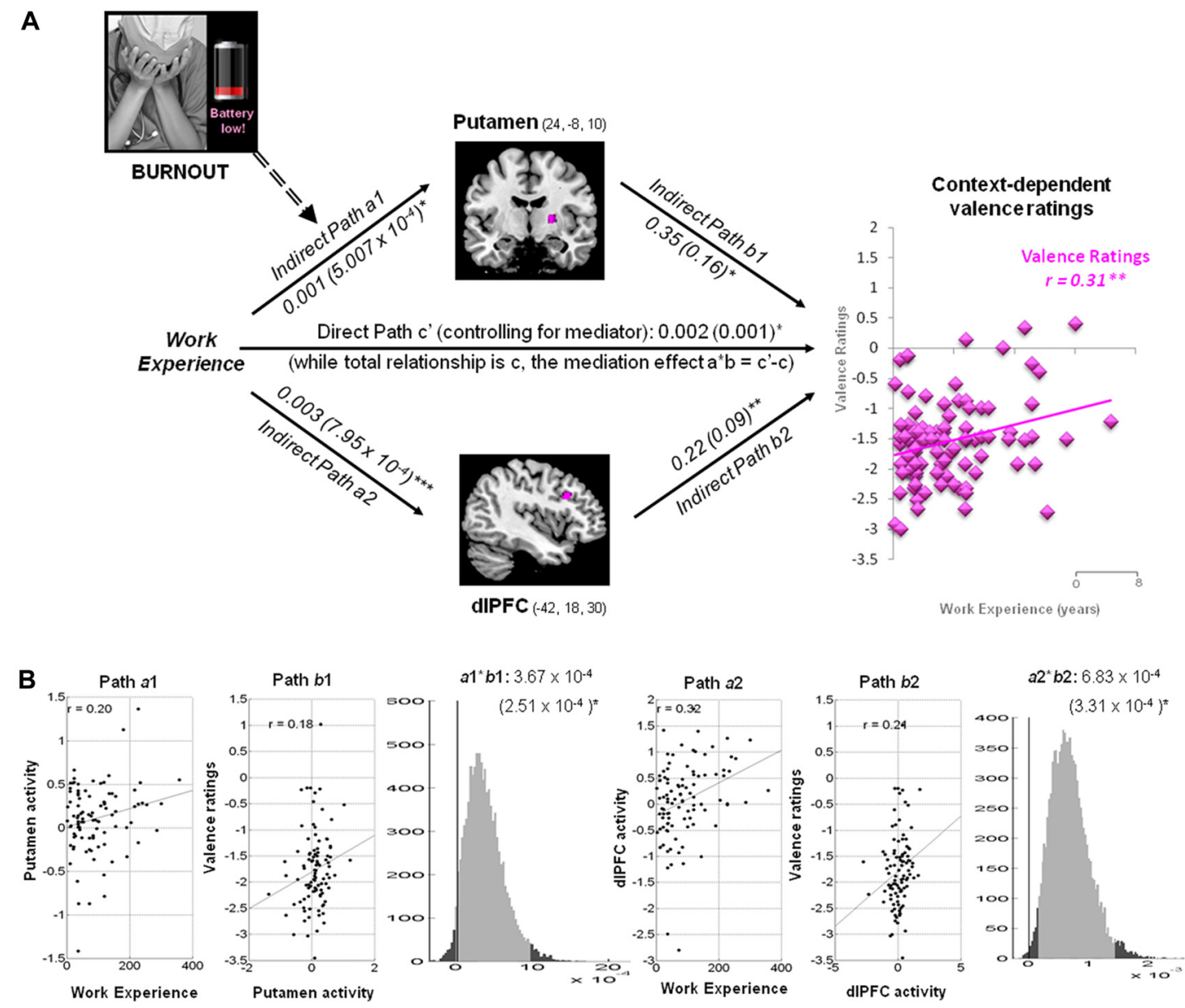

C Total relationship between Work Experience and Putamen activity
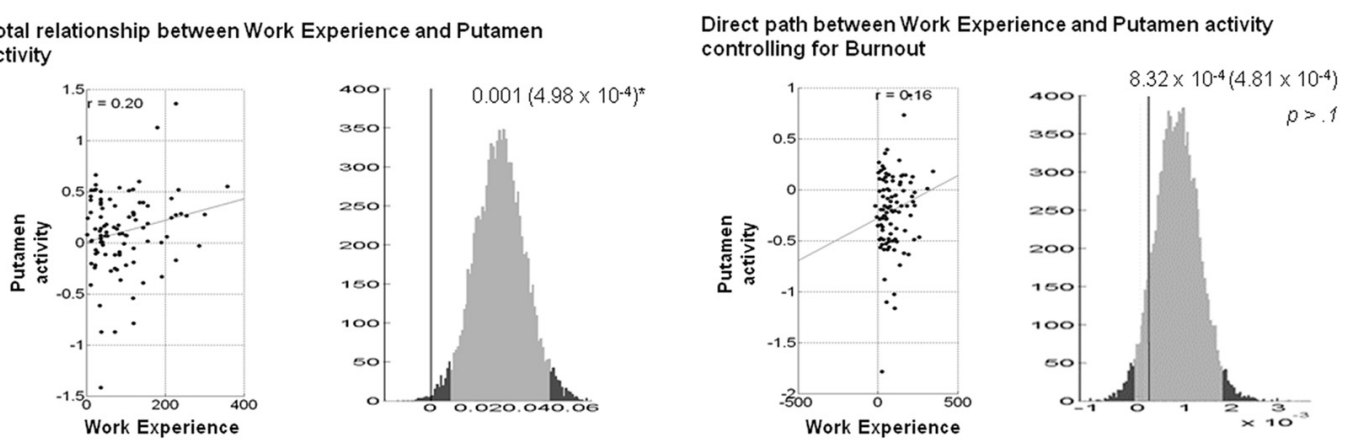

FIGURE 4 | Mediation analysis of context-dependent valence ratings. (A) Path diagram demonstrates the relationship between variables in the path model. Work experience (left) as the predictor variable predicts the hemodynamic activity in the putamen (top) and dIPFC (bottom). The connection of work experience to each brain mediator (putamen or dIPFC) is the a path. The lines are labeled with path coefficients, and standard errors are shown in parentheses. The connection of each mediator to the outcome (valence ratings) is the $b$ path. They are calculated controlling for work experience and for other mediators, as the standard in mediation models. The inclusion of burnout levels into the mediation model abolishes the causal relationship between work experience (left panel), putamen activity (top), and context-dependent valence ratings (right panel). Scores on reduced personal accomplishment, as one of burnout constructs, moderates the mediation effect of putamen (path a1, dash arrow). ${ }^{* * *} p<0.001,{ }^{* *} p<0.01,{ }^{*} p<0.05$, two-tailed. The direct path is the $c^{\prime}$ path, which is calculated controlling for both mediators. (B) Substantiation of the mediation path $a, b$ and $c$. Regression scatterplots depict the relationships between predictor (i.e., working experience) and ROls (path a1 and a2). Partial regression scatterplots demonstrate the relationships between ROls (putamen and dIPFC) and valence ratings (path b1 and b2). The mediation effect $\left(a 1^{*} b 1\right.$ and $\left.a 2 * b 2\right)$ is substantiated by the bootstrapped distributions. The range on the $x$-axis spanned by the lighter gray portion of the histogram is the $95 \%$ confidence interval for the effect. (C) A significant correlation between work experience and putamen (path a1) was found before Burnout being included into the mediation model (left panel). One of the Burnout subscales, reduced personal accomplishment, affected achievement-motivational processing in the putamen and modulated the mediation effect of putamen (path a1), thus abolish the causal relationship between work experience, putamen and valence processing in hospital context (right panel). 
A
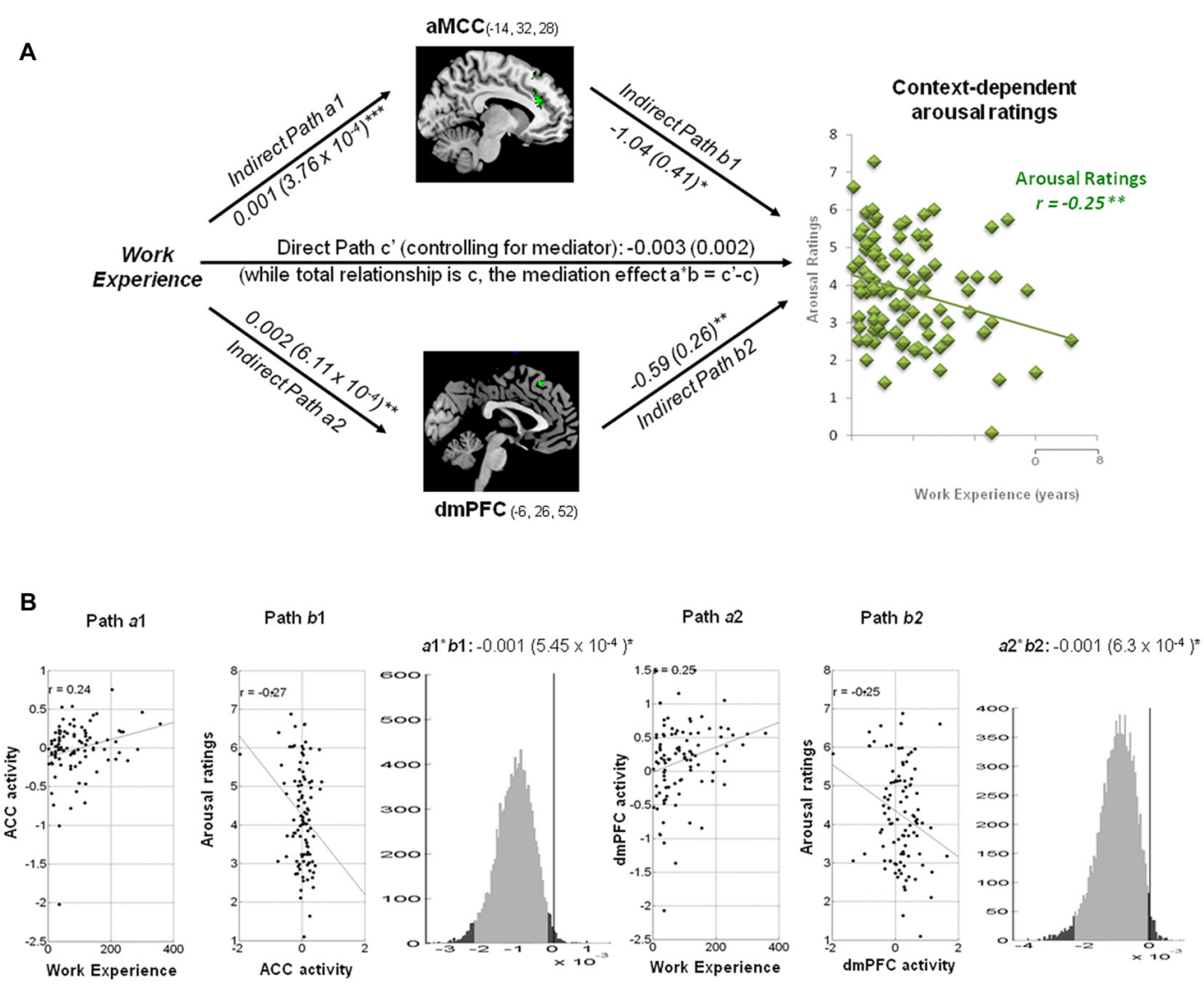

FIGURE 5 | Mediation analysis on context-dependent arousal ratings. (A) Path diagram shows the relationship between variables in the path model. Work experience (left) as the predictor variable predicts the activity in the ACC (top) and dmPFC (bottom). The connection of work experience to each brain mediator (ACC or dmPFC) is the a path. The lines are labeled with path coefficients, and standard errors are shown in parentheses. The connection of each mediator to the outcome (arousal ratings) is the $b$ path. They are calculated controlling for work experience and for other mediators, as the standard in mediation models. ${ }^{* * *} p<0.001,{ }^{* *} p<0.01,{ }^{*} p<0.05$, two-tailed. The direct path is the $c^{\prime}$ path, which is calculated controlling for both mediators. (B) Substantiation of the mediation path $a, b$ and $c$. Regression scatterplots demonstrate the relationships between predictor (work experience) and ROls (path a1 and a2). Partial regression scatterplots depict the relationships between ROls (ACC and dmPFC) and arousal ratings (path $b 1$ and $b 2)$. The mediation effect (a1*b1 and a2*b2) is substantiated by the bootstrapped distributions. The range on the $x$-axis spanned by the lighter gray portion of the histogram is the $95 \%$ confidence interval for the effect. None of each Burnout subscale modulated the mediation effect of arousal ratings.

responses when viewing others in pain are influenced by their work experience and situational context.

In spite of the close association between work experience and age $(r=0.95)$, the context-dependent subjective ratings of the painful stimuli cannot be explained by the aging process itself. One previous study using similar stimuli did not find any age-related changes in subjective ratings in individuals between 20 years old and 80 years old (Chen et al., 2014). Measures of dispositional empathy throughout the adult lifespan showed an inverse-U-shaped pattern across age (O'Brien et al., 2013). In the present study, nurses' work experience (in year) had a linear effect on the subjective ratings and burnout levels. Medical practitioners who have burnout might tend to change their career path.

Interestingly, nurses with longer work experience evaluated pain stimuli in a hospital context as less negative in both valence and arousal. They also reported less depersonalization and better personal work accomplishment compared with less experience nurses. Experienced nurses may have higher quality and more cost-effective care (Bartel et al., 2014). Contextdependent subjective ratings in relation to work experience may reflect achievement-related processes that modulate motivation to empathy-eliciting stimuli (Takeuchi et al., 2014).

Consistent with previous fMRI studies of pain empathy (Lamm et al., 2011 for a meta-analysis), significant hemodynamic activity was detected in the pain matrix when the nurses perceived pain stimuli (see Table 1). In addition, 25 participant controls included in this study also showed a similar pattern of neuro-hemodynamic responses (Supplementary Table S1). One previous study showed that the anterior insula and aMCC were significantly activated in the controls, but not in the physicians, who instead showed activation of the rTPJ (Cheng et al., 2007). The present study extends these results by demonstrating that the home context led to a stronger activation in the insula and aMCC 
than did the hospital context, whereas the reverse comparison showed increased activation in the rTPJ.

In a hospital context, rather than a home context, the insula elicited by pain stimuli revealed a negative functional connectivity with the vmPFC, and a positive coupling with the MCC. A neuroimaging study with physicians found a negative covariation of the $\mathrm{mPFC}$ with the insula in response to viewing patients being pricked by needles (Cheng et al., 2007). The negative connectivity of the insula with the mPFC elicited by painful stimuli could reflect a cognitive inhibition of their affective processing to patient's distress, as the mPFC is involved in ToM and emotion regulation (Cheng et al., 2010; Etkin et al., 2011; Zaki and Ochsner, 2012). This finding is consistent with a detached perspective adopted by some medical practitioners. The detachment, orchestrated by executive function, blunts potential emotional contagion from patients' suffering and allows medical practitioners to respond to patients with adequate assistance.

Importantly, the two situational contexts produced distinct associations between the subjective ratings of valence and the neurohemodynamic response to perceiving painful stimuli. The negative valence ratings of pain in a home context were positively coupled with enhanced activity in the insula. The hospital context, however, led to more positive valence ratings and predicted increased activity in the putamen and dlPFC. It can be argued that medical practitioners must not become detached and callous except when working in a hospital. The neuro-hemodynamic response in the insula, a region that plays a key role in empathy by mapping internal states of bodily and subjective feelings, suggests that medical practitioners are more emotionally engaged when the pain is perceived in the home context (Banissy et al., 2012). On the other hand, the putamen, as part of the striatum, is involved in the processing of reward (O'Doherty et al., 2002; Mizuno et al., 2008). The dlPFC is implicated in executive control of negative emotions (Ochsner et al., 2004) and emotion regulation of empathy (Decety, 2011). Healthcare involves neural representations of treatment expectation, reward and empathy (Jensen et al., 2014). The response in the putamen and dlPFC reflects how medical training and expertise enables medical practitioners to dissociate from vicariously sharing patients' suffering and thus from becoming overwhelmed by negative emotions. Furthermore, in addition to situational context, interpersonal variables, implicit attitudes and group preferences could either strengthen or weaken the neural network implicated in empathy for pain (Decety and Cowell, 2014). Here, to probe the effect specific to situational context, participants were instructed to imagine to face the same person (for example, a friend or family member) who was in the pain/no-pain in both of home and hospital contexts.

Mediation analyses indicate that reduced personal accomplishments, one aspect of burnout, moderates the mediation effect of putamen activity on context-dependent valence ratings. Burnout as a multidimensional process includes emotional exhaustion, depersonalization and reduced personal accomplishment (Maslach and Jackson, 1981). Nurses with higher dispositional empathy were more prone to emotional exhaustion, which was associated with lower activation in the insula and rTPJ (Tei et al., 2014). Our findings also demonstrate that nurses with more burnout symptoms were less likely to have longer work experience and to give positive valence ratings. Nurses with more burnout symptoms also showed less achievement- related putamen activation when viewing pain in a hospital context. These findings support the notion that perceiving reward from patient care can protect medical practitioners from burnout (Basińska and Wilczek-Ruyczka, 2013).

Some limitations of this study must be acknowledged. First, co-effects of expectations of the nurses with respect to hospital and home contexts might contribute to contextual effect for empathic neural responses. Second, the studies on occupational wellbeing in nurses have shown significant associations between coping strategies, personality characteristics, burnout and clinical performance (Adriaenssens et al., 2015; CañadasDe la Fuente et al., 2015). High neuroticism with the opposite of emotional stability tends to use non-effective coping strategies, such as, avoidance and distraction, and can lead to higher levels of burnout. Hence, future studies to address the interplay between working experience, coping strategies and neural underpinning of pain empathy are warranted.

\section{CONCLUSION}

Contextual modulation of empathy represents an adaptive advantage, making behavior more sensitive to different environment conditions (Melloni et al., 2014). A better understanding of how situational context influences the neurocognitive mechanisms underpinning emotional sensitivity and empathy in medicine can contribute to preventing serious health hazards and risks in medical professionals. It also sheds light on the challenge to achieve an appropriate balance between empathy and clinical distance. Detachment is often seen as necessary not only to avoid burning out or losing control, as well as allow practitioners to provide objective medical care. Our results showed that, instead of generalization of desensitizing effect of others' pain and suffering outside of the workplace, healthcare experience did not hamper nurses' motivation to detect the pain of another person at home context. Working experience with less depersonalization and better personal work accomplishment could shape the brain of medical professions in the way to offer healthcare benefits to both caregivers and patients. Switching perspectives between hospital and home contexts might help medical professions release from workplace stress. Of note, perceiving rewards from patient care protects medical practitioners from burnout.

\section{AUTHOR CONTRIBUTIONS}

YC and JD conceived and designed the experiments, wrote the first draft of the manuscript. CC and YC collected the data, contributed to data analysis. All authors contributed to data interpretation and manuscript write-up. 


\section{ACKNOWLEDGMENTS}

We thank Ya-Han Chou for data collection. The study was funded by the Ministry of Science and Technology (MOST 1032410-H-010-003-MY3; 106-2420-H-010-004-MY2; 106-2410H-010-002-MY2), National Yang-Ming University Hospital (RD2017-005) and Ministry of Education (Aim for the Top University Plan). Dr. Jean Decety was supported by a grant

\section{REFERENCES}

Adriaenssens, J., De Gucht, V., and Maes, S. (2015). Determinants and prevalence of burnout in emergency nurses: a systematic review of 25 years of research. Int. J. Nurs. Stud. 52, 649-661. doi: 10.1016/j.ijnurstu.2014.11.004

Atlas, L. Y., Bolger, N., Lindquist, M. A., and Wager, T. D. (2010). Brain mediators of predictive cue effects on perceived pain. J. Neurosci. 30, 12964-12977. doi: 10.1523/JNEUROSCI.0057-10.2010

Baez, S., and Ibanez, A. (2014). The effects of context processing on social cognition impairments in adults with Asperger's syndrome. Front. Neurosci. 8:270. doi: 10.3389/fnins.2014.00270

Bagby, R. M., Parker, J. D. A., and Taylor, G. J. (1994). The twenty-item Toronto Alexithymia Scale-I. Item selection and cross-validation of the factor structure. J. Psychosom. Res. 38, 23-32. doi: 10.1016/0022-3999(94)90005-1

Banissy, M. J., Kanai, R., Walsh, V., and Rees, G. (2012). Inter-individual differences in empathy are reflected in human brain structure. Neuroimage 62, 2034-2039. doi: 10.1016/j.neuroimage.2012.05.081

Baron, R. M., and Kenny, D. A. (1986). The moderator-mediator variable distinction in social psychological research: conceptual, strategic, and statistical considerations. J. Pers. Soc. Psychol. 51, 1173-1182. doi: 10.1037/0022-3514.51. 6.1173

Bartel, A. P., Beaulieu, N. D., Phibbs, C. S., and Stone, P. W. (2014). Human capital and productivity in a team environment: evidence from the healthcare sector. Am. Econ. J. Appl. Econ. 6, 231-259. doi: 10.1257/app.6.2.231

Basińska, B. A., and Wilczek-Ruzyczka, E. (2013). The role of rewards and demands in burnout among surgical nurses. Int. J. Occup. Med. Environ. Health 26, 593-604. doi: 10.2478/s13382-013-0129-8

Berntson, G. G., Norman, G. J., Bechara, A., Bruss, J., Tranel, D., and Cacioppo, J. T. (2011). The insula and evaluative processes. Psychol. Sci. 22, 80-86. doi: 10.1177/0956797610391097

Bzdok, D., Heeger, A., Langner, R., Laird, A. R., Fox, P. T., PalomeroGallagher, N., et al. (2015). Subspecialization in the human posterior medial cortex. Neuroimage 106, 55-71. doi: 10.1016/j.neuroimage.2014.11.009

Bzdok, D., Langner, R., Schilbach, L., Jakobs, O., Roski, C., Caspers, S., et al. (2013). Characterization of the temporo-parietal junction by combining data-driven parcellation, complementary connectivity analyses, and functional decoding. Neuroimage 81, 381-392. doi: 10.1016/j.neuroimage.2013.05.046

Cañadas-De la Fuente, G. A., Vargas, C., San Luis, C., García, I., Cañadas, G. R., and De la Fuente, E. I. (2015). Risk factors and prevalence of burnout syndrome in the nursing profession. Int. J. Nurs. Stud. 52, 240-249. doi: 10.1016/j.ijnurstu. 2014.07.001

Chen, Y.-C., Chen, C.-C., Decety, J., and Cheng, Y. (2014). Aging is associated with changes in the neural circuits underlying empathy. Neurobiol. Aging 35, 827-836. doi: 10.1016/j.neurobiolaging.2013.10.080

Chen, C., Yang, C. Y., and Cheng, Y. (2012). Sensorimotor resonance is an outcome but not a platform to anticipating harm to others. Soc. Neurosci. 7, 578-590. doi: 10.1080/17470919.2012.686924

Cheng, Y., Chen, C., Lin, C. P., Chou, K. H., and Decety, J. (2010). Love hurts: an fMRI study. Neuroimage 51, 923-929. doi: 10.1016/j.neuroimage.2010. 02.047

Cheng, Y., Lin, C. P., Liu, H. L., Hsu, Y. Y., Lim, K. E., Hung, D., et al. (2007). Expertise modulates the perception of pain in others. Curr. Biol. 17, 1708-1713. doi: 10.1016/j.cub.2007.09.020

Davis, M. H. (1996). Empathy: A Social Psychological Approach. Madison, WI: Westview Press.

Decety, J. (2011). Dissecting the neural mechanisms mediating empathy. Emot. Rev. 3, 92-108. doi: 10.1177/1754073910374662 from the John Templeton Foundation (Wisdom Research at the University of Chicago).

\section{SUPPLEMENTARY MATERIAL}

The Supplementary Material for this article can be found online at: http://journal.frontiersin.org/article/10.3389/fnbeh.2017.001 65/full\#supplementary-material

Decety, J. (2015). The neural pathways, development and functions of empathy. Curr. Opin. Behav. Sci. 3, 1-6. doi: 10.1016/j.cobeha.2014.12.001

Decety, J., Chen, C., Harenski, C., and Kiehl, K. A. (2013). An fMRI study of affective perspective taking in individuals with psychopathy: imagining another in pain does not evoke empathy. Front. Hum. Neurosci. 7:489. doi: 10.3389/fnhum.2013.00489

Decety, J., and Cowell, J. M. (2014). The complex relation between morality and empathy. Trends Cogn. Sci. 18, 337-339. doi: 10.1016/j.tics.2014.04.008

Decety, J., Echols, S., and Correll, J. (2010a). The blame game: the effect of responsibility and social stigma on empathy for pain. J. Cogn. Neurosci. 22, 985-997. doi: 10.1162/jocn.2009.21266

Decety, J., Yang, C. Y., and Cheng, Y. (2010b). Physicians down-regulate their pain empathy response: an event-related brain potential study. Neuroimage 50, 1676-1682. doi: 10.1016/j.neuroimage.2010.01.025

Decety, J., and Jackson, P. L. (2004). The functional architecture of human empathy. Behav. Cogn. Neurosci. Rev. 3, 71-100. doi: $10.1177 / 1534582304267187$

Decety, J., Smith, K. E., Norman, G. J., and Halpern, J. (2014). A social neuroscience perspective on clinical empathy. World Psychiatry 13, 233-237. doi: 10.1002/wps.20146

Dehning, S., Girma, E., Gasperi, S., Meyer, S., Tesfaye, M., and Siebeck, M. (2012). Comparative cross-sectional study of empathy among first year and final year medical students in Jimma University, Ethiopia: steady state of the heart and opening of the eyes. BMC Med. Educ. 12:34. doi: 10.1186/1472-69 20-12-34

Dyrbye, L. N., and Shanafelt, T. D. (2011). Physician burnout: a potential threat to successful health care reform. JAMA 305, 2009-2010. doi: 10.1001/jama.20 11.652

Etkin, A., Egner, T., and Kalisch, R. (2011). Emotional processing in anterior cingulate and medial prefrontal cortex. Trends Cogn. Sci. 15, 85-93. doi: 10.1016/j.tics.2010.11.004

Figley, C. R. (1995). "Compassion fatigure: toward a new understanding of the costs of caring," in Secondary Traumatic Stress: Self-Care Issues for Clinicians, Researchers, and Educators, ed. B. H. Stamm (Lutherville, MD: Sidran Press), 3-28.

Figley, C. R. (2012). "The empathic response in clinical practice: antecedents and consequences," in Empathy: From Bench to Bedside, ed. J. Decety (Cambridge, MA: MIT Press), 263-273.

Franck, L. S., and Bruce, E. (2009). Putting pain assessment into practice: why is it so painful? Pain Res. Manag. 14, 13-20. doi: 10.1155/2009/ 856587

Gleichgerrcht, E., and Decety, J. (2012). "The costs of empathy among health professionals," in Empathy: From Bench to Bedside, ed. J. Decety (Cambridge, MA: MIT Press), 245-261.

Gleichgerrcht, E., and Decety, J. (2014). The relationship between different facets of empathy, pain perception and compassion fatigue among physicians. Front. Behav. Neurosci. 8:243. doi: 10.3389/fnbeh.2014.00243

Halpern, J. (2003). What is clinical empathy? J. Gen. Intern. Med. 18, 670-674. doi: 10.1046/j.1525-1497.2003.21017.x

Halpern, J. (2012). "Clinical empathy in medical care," in Empathy: From Bench to Bedside, ed. J. Decety (Cambridge, MA: MIT Press), 229-244.

Hasselhorn, H. M., Tackenberg, P., and Peter, R. (2004). Effort-reward imbalance among nurses in stable countries and in countries in transition. Int. J. Occup. Environ. Health 10, 401-408. doi: 10.1179/oeh.2004.10.4.401

Jackson, P. L., Rainville, P., and Decety, J. (2006). To what extent do we share the pain of others? Insight from the neural bases of pain empathy. Pain 125, 5-9. doi: 10.1016/j.pain.2006.09.013 
Jensen, K. B., Petrovic, P., Kerr, C. E., Kirsch, I., Raicek, J., Cheetham, A., et al. (2014). Sharing pain and relief: neural correlates of physicians during treatment of patients. Mol. Psychiatry 19, 392-398. doi: 10.1038/mp.2012.195

Kappesser, J., and Williams, A. C. (2002). Pain and negative emotions in the face: judgements by health care professionals. Pain 99, 197-206. doi: 10.1016/s03043959(02)00101-x

Lamm, C., Decety, J., and Singer, T. (2011). Meta-analytic evidence for common and distinct neural networks associated with directly experienced pain and empathy for pain. Neuroimage 54, 2492-2502. doi: 10.1016/j.neuroimage. 2010.10.014

Lin, Y.-C., and Chan, C.-H. (2003). A factor analysis of the taiwan version of the toronto alexithymia scale-20. Taiwan. J. Psychiatry 17, 276-282.

Lindquist, M. A. (2012). Functional causal mediation analysis with an applicatin to brain connectivity. J. Am. Stat. Assoc. 107, 1297-1309. doi: 10.1080/01621459. 2012.695640

MacKinnon, D. P., Fairchild, A. J., and Fritz, M. S. (2007). Mediation analysis. Annu. Rev. Psychol. 58, 593-614. doi: 10.1146/annurev.psych.58.110405.085542

Maslach, C., and Jackson, S. (1981). The measurement of experienced burnout. J. Occup. Behav. 2, 99-113. doi: 10.1002/job.4030020205

Maslach, C., Jackson, S. E., and Leiter, M. P. (1996). Maslach Burnout Inventory. Palo Alto, CA: Consulting Psychologists Press.

Melloni, M., Lopez, V., and Ibanez, A. (2014). Empathy and contextual social cognition. Cogn. Affect. Behav. Neurosci. 14, 407-425. doi: 10.3758/s13415-0130205-3

Menon, V., and Uddin, L. Q. (2010). Saliency, switching, attention and control: a network model of insula function. Brain Struct. Funct. 214, 655-667. doi: 10.1007/s00429-010-0262-0

Mizuno, K., Tanaka, M., Ishii, A., Tanabe, H. C., Onoe, H., Sadato, N., et al. (2008). The neural basis of academic achievement motivation. Neuroimage 42, 369-378. doi: 10.1016/j.neuroimage.2008.04.253

Morrison, S., Decety, J., and Molenberghs, P. (2012). The neuroscience of group membership. Neuropsychologia 50, 2114-2120. doi: 10.1016/j. neuropsychologia.2012.05.014

Nielsen, H. G., and Tulinius, C. (2009). Preventing burnout among general practitioners: is there a possible route? Educ. Prim. Care 20, 353-359. doi: 10.1080/14739879.2009.11493817

Norris, C. J., Gollan, J., Berntson, G. G., and Cacioppo, J. T. (2010). The current status of research on the structure of evaluative space. Biol. Psychol. 84, 422-436. doi: 10.1016/j.biopsycho.2010.03.011

O’Brien, E., Konrath, S. H., Grühn, D., and Hagen, A. L. (2013). Empathic concern and perspective taking: linear and quadratic effects of age across the adult life span. J. Gerontol. B Psychol. Sci. Soc. Sci. 68, 168-175. doi: 10.1093/geronb/gbs055

Ochsner, K. N., Ray, R. D., Cooper, J. C., Robertson, E. R., Chopra, S., Gabrieli, J. D., et al. (2004). For better or for worse: neural systems supporting the cognitive down- and up-regulation of negative emotion. Neuroimage 23, 483-499. doi: 10.1016/j.neuroimage.2004.06.030
O’Doherty, J. P., Deichmann, R., Critchley, H. D., and Dolan, R. J. (2002). Neural responses during anticipation of a primary taste reward. Neuron 33, 815-826. doi: 10.1016/s0896-6273(02)00603-7

Preis, M. A., Schmidt-Samoa, C., Dechent, P., and Kroener-Herwig, B. (2013). The effects of prior pain experience on neural correlates of empathy for pain: an fMRI study. Pain 154, 411-418. doi: 10.1016/j.pain.2012.11.014

Riess, H. (2015). The impact of clinical empathy on patients and clinicians: understanding empathy's side effects. AJOB Neurosci. 6, 51-53. doi: 10.1080/21507740.2015.1052591

Schilbach, L. (2014). On the relationship of online and offline social cognition. Front. Hum. Neurosci. 8:278. doi: 10.3389/fnhum.2014.00278

Silani, G., Lamm, C., Ruff, C. C., and Singer, T. (2013). Right supramarginal gyrus is crucial to overcome emotional egocentricity bias in social judgments. J. Neurosci. 33, 15466-15476. doi: 10.1523/JNEUROSCI.1488-13.2013

Takeuchi, H., Taki, Y., Nouchi, R., Sekiguchi, A., Kotozaki, Y., Miyauchi, C. M., et al. (2014). Regional gray matter density is associated with achievement motivation: evidence from voxel-based morphometry. Brain Struct. Funct. 219 , 71-83. doi: 10.1007/s00429-012-0485-3

Tei, S., Becker, C., Kawada, R., Fujino, J., Jankowski, K. F., Sugihara, G., et al. (2014). Can we predict burnout severity from empathy-related brain activity? Transl. Psychiatry 4:e393. doi: 10.1038/tp.2014.34

Wager, T. D., Davidson, M. L., Hughes, B. L., Lindquist, M. A., and Ochsner, K. N. (2008). Prefrontal-subcortical pathways mediating successful emotion regulation. Neuron 59, 1037-1050. doi: 10.1016/j.neuron.2008.09.006

Xavier Balda, R., Guinsburg, R., de Almeida, M. F., Peres, C., Miyoshi, M. H., and Kopelman, B. I. (2000). The recognition of facial expression of pain in full-term newborns by parents and health professionals. Arch. Pediatr. Adolesc. Med. 154, 1009-1016. doi: 10.1001/archpedi.154.10.1009

Yang, C. Y., Decety, J., Lee, S., Chen, C., and Cheng, Y. (2009). Gender differences in the mu rhythm during empathy for pain: an electroencephalographic study. Brain Res. 1251, 176-184. doi: 10.1016/j.brainres.2008.11.062

Zaki, J., and Ochsner, K. N. (2012). The neuroscience of empathy: progress, pitfalls and promise. Nat. Neurosci. 15, 675-680. doi: 10.1038/nn.3085

Zenasni, F., Boujut, E., Woerner, A., and Sultan, S. (2012). Burnout and empathy in primary care: three hypotheses. Br. J. Gen. Pract. 62, 346-347. doi: 10.3399/bjgp12x652193

Conflict of Interest Statement: The authors declare that the research was conducted in the absence of any commercial or financial relationships that could be construed as a potential conflict of interest.

Copyright (C) 2017 Cheng, Chen and Decety. This is an open-access article distributed under the terms of the Creative Commons Attribution License (CC BY). The use, distribution or reproduction in other forums is permitted, provided the original author(s) or licensor are credited and that the original publication in this journal is cited, in accordance with accepted academic practice. No use, distribution or reproduction is permitted which does not comply with these terms. 